\title{
THE USE OF PROTEOMIC TECHNOLOGIES IN BREAST CANCER RESEARCH
}

\author{
M.G. Mazur*, T.V. Pyatchanina \\ R.E. Kavetsky Institute of Experimental Pathology, Oncology and Radiobiology, NASof Ukraine, Kyiv 03022, Ukraine
}

The main findings in the field of breast cancer proteomic research as well as modern strategies, technologies and methods of validation are reviewed. A special attention is focused on validated proteomic biomarkers of breast cancer. The data on proteomic profiling of stroma, tumor microenvironment, involvement of proteins in tumor progression, invasion and metastasis, and mechanisms of action of new generation drugs, are analyzed. The results of proteomic analysis are of high clinical importance and significantly improve tumor molecular profiling, stratification of patients, screening, diagnostics, and therapy of breast cancer.

Key Words: breast cancer, proteomic biomarkers of breast cancer, proteomic technologies, strategy and methods for identification of proteins, MS-based methods.

Breast cancer $(\mathrm{BC})$ is a highly complex systemic disease with different histological forms and molecular subtypes. The biologic complexity of BC is determined by significant intratumoral heterogeneity that is characterized by physiologic, morphologic, molecular, genetic and epigenetic features. The development of the strategy for personalized approach for diagnostics and therapy of BC patients requires advanced knowledge

Submitted: August 05, 2016.

${ }^{*}$ Correspondence: E-mail: mariamazur17@gmail.com

Phone: +38 (098) 994-70-39

Abbreviations used: 2D-DIGE - two-dimensional differential in-gel electrophoresis; 2D-PAGE - 2D-polyacrylamide gel electrophoresis; APE1 - apurinic/apyrimidinic endonuclease 1; BC - breast cancer; CAFs - cancer-associated fibroblasts; EPR - endoplasmic reticulum; ER - estrogen receptor; ESI - electrospray ionization source; FFPA - forward phase protein array; FFPE - formalin-fixed, paraffinembedded; FT-ICR MS - fourier transform ion cyclotron resonance analyzer of mass spectrometry; HER2 - human epidermal growth factor receptor 2; ICAT - isotope-coded affinity tags; IHC - immunohistochemical analysis; iTRAQ - isobaric tags for relative and absolute quantification; KIFAP3 - kinesin associated protein 3; LC - liquid chromatography; LCM - laser capture microdissection; LTQ-Orbitrap MS - hybrid linear ion trap analyzer of mass spectrometry; MALDI matrix-assisted laser desorption-ionization; MD-LC - multidimensional liquid chromatography; MRM-MS - multiple reaction monitoring mass spectrometry; MS - mass spectrometry; MS/MS - tandem mass spectrometry; mTRAQ - mass differential tags for relative and absolute quantification; MudPIT - multidimensional protein identification technology; Nanospray MS - ionization source of mass spectrometry; $\mathrm{nLC}-\mathrm{MS} / \mathrm{MS}$ - nanoscale liquid chromatography-tandem mass spectrometry; PR - progesterone receptor; Q MS - quadrupoles analyzer of mass spectrometry; Q-Orbitrap MS - hybrid analyzer of mass spectrometry; Q-TOF MS - hybrid quadrupoles-time-of-flight analyzer of mass spectrometry; RP-LC - reverse phase liquid chromatography; RPPA - reversed phase protein array; RRBP1 - ribosome binding protein 1; SAX - strong anionic exchange; SCX - strong cationic exchange; SDS - sodium dodecyl sulphate; SELDI - surface enhanced laser desorption/ionization source; SILAC - stable isotope labeling by amino acids in cell culture; SRM-MS - selective reaction monitoring mass spectrometry; SW - software; TMA - tissue microarrays; TNBC triple-negative breast cancer subtype; TOF - time-of-flight analyzer; TripleTOF MS - hybrid time-of-flight analyzer of mass spectrometry; XCT II MS (TripleQ MS) - hybrid quadrupoles analyzer of mass spectrometry. on molecular markers of malignant transformation and treatment response for improvement of diagnostic tests, survival indexes and quality of life of the patients, and the development of new generation anticancer therapeutics [1-3].

The studies of BC proteome are driven foremost by the necessity for an analysis of information accumulated within the frameworks of "Human Genome" project at the levels of trascriptome, proteome, and metabolome. The relation between proteome with other areas of functional genomics is presented in Fig. 1 [4]. When immunohistochemical analysis $(\mathrm{IHC})$ has been introduced into clinical practice as a diagnostic method, it became possible to study the state of specific protein receptors in $B C$ patients, and, consequently, to identify the molecular subtypes of $B C$ such as luminal $A$ and $B$, basal, Her2-expressing subtype, and subtype histologically similar to normal phenotype $[5,6]$. In turn, proteome and interactome of the molecular BC subtypes are highly heterogeneous, therefore the clinical use of personalized therapy presupposes an identification of protein markers for diagnostics of $\mathrm{BC}$ and the disease prognosis. Despite the newest achievements in the field of genetic and histological assays, the deficit of molecular diagnostic methods for determination of BC features is still evident [6].

The present review is devoted to an analysis of modern strategies, technologies and scientific findings in proteomic research of BC.

Proteomic strategies, technologies and study subject. In recent years, a number of studies were directed on the determination of relevant panel of protein markers of $\mathrm{BC}$ molecular subtypes matching the criteria for standard clinical study of patient's biologic material for diagnostics, prognosis and therapy. Along with this, the development of protein profile and identification of protein biomarkers of $\mathrm{BC}$ in body tissues and fluids (classification of biomarkers [7], Fig. 2) should meet the requirements, in particular, for high level of reproducibility in different laboratories during an analysis of monotypic material. In parallel with increasing bulk of experimental findings potentially important for clinical practice, there has been performed an improvement 


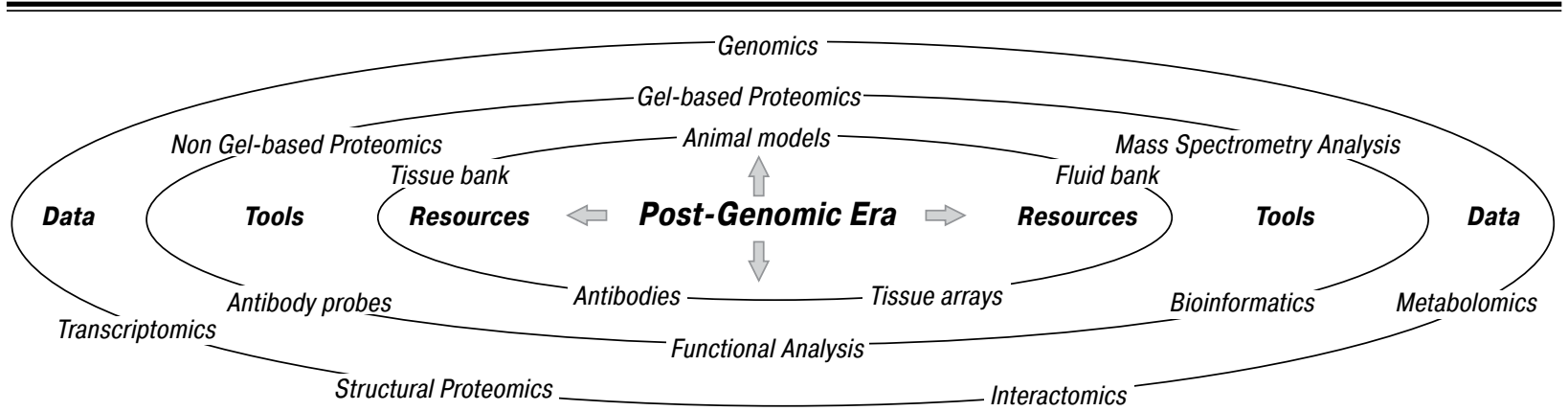

Fig. 1. Interrelation of sources, technologies, and "omics" data in proteomic studies of BC

of existing approaches and analytic methods for protein research and technical capabilities of equipment, in particular, via combination of a few simple methods and devices.

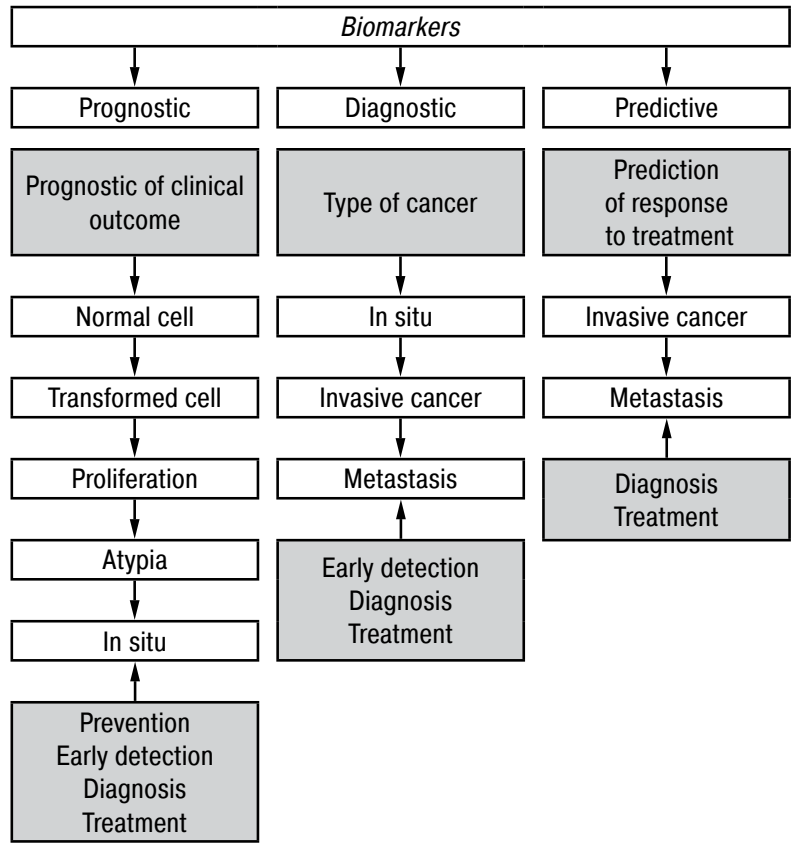

Fig. 2. Classification of biomarkers by their assignment and relation to tumor progression

Strategies. Advantages and limitations of the strategies for detection of cancer biomarkers are reviewed in detail in [8] (Fig. 3).

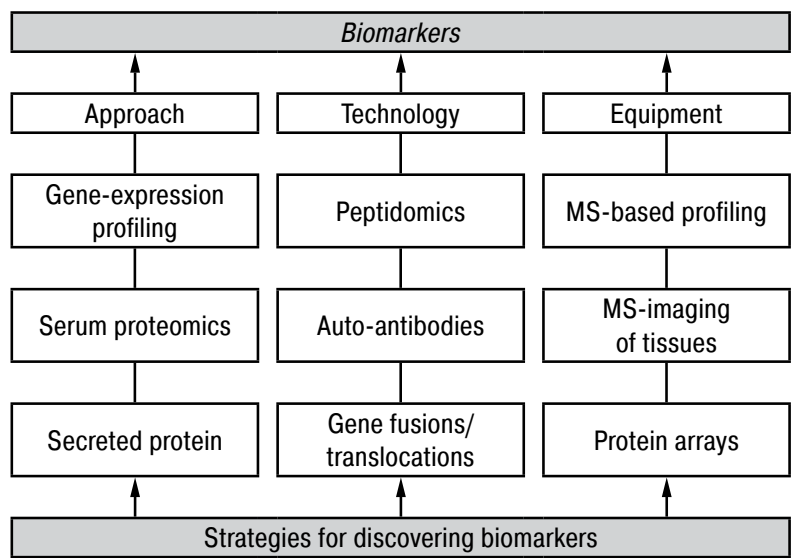

Fig. 3. Classification of strategies for discovering BC biomarkers

The known strategies of preparing the samples for protein profiling, so called "bottom-up analysis" and "top-down analysis" are principally different at an initial stage of a sample treatment and are used in the methods based on gel-electrophoresis, liquid chromatography (LC) and mass spectrometry (MS) [9]. Bottom-up analysis requires initial enzymatic digestion of protein molecules into peptides with the use of proteases. The strategy is used in a few cases: firstly, for identification of proteins through peptide analysis with their following search in databases; secondly, for chemical modification of the peptides for quantification of peptides and proteins. Top-down analysis deals with intact preparations where protein molecules remain undamaged and undigested, and is used for an analysis of separate proteins or simple protein mixes, an analysis of protein-protein complexes and target proteins, or for multiple identification of proteins with post-translational modifications. The main advantages and disadvantages of these strategies are as follows [9]:

- bottom-up analysis allows one to analyze the samples of high complexity, provides a set of large data bases, is more sensitive; however, it requires repeated analysis of the samples with large peptide variability, is limited by protein sequence coverage by identified peptides, is ambiguitous regarding the origin for redundant peptide sequences;

- top-down analysis allows one to identify isoforms of proteins and study labile proteins with posttranslational modifications, improves quantification but has the limitations associated with precursor ion charge state resulting in some problems in analyzing proteins with charge-state ambiguity and front-end separation (as far as the range of methods for protein separation is limited).

Methodological instruments for the use of mentioned strategies are various: in proteomic studies performed by bottom-up strategy, such methods as gel electrophoresis, affine chromatography (including isotope-coded affinity tags - ICAT), ion-exchange chromatography, reverse phase liquid chromatography (RP-LC), Q-TOF MS, LTQ-Orbitrap MS are used, while top-down analysis studies exploit the methods of ion-exchange chromatography, RP-LC, 2D-LC, ESI MS, LTQ-Orbitrap MS [9]. Some methods, for example, RP-LC та LTQ-Orbitrap MS, could be used for both strategies.

Technologies. Different classifications of technologies for proteomic studies that are used for an analysis of tumor tissues and body fluids are known [10]. The methods of proteomic studies are based on the use of antibodies, in particular, Western blot, enzyme-linked 
immunosorbent assay (ELISA), IHC, tissue microarray (TMA), forward phase protein array (FFPA) and reverse phase protein array (RPPA), or these methods are not exploiting antibodies and are based on MS. The first group of methods is used for verification and validation of the obtained data for further use of the results in clinical practice and requires an established knowledge of the proteins under study, while the second group of methods is represents the experimental platforms for generation of databases for identified proteins.

By the type of equipment used in the research, one may classify the proteomic technologies as follows: methods of gel electrophoresis (2D-PAGE, 2D-DIGE), peptide-oriented proteomics (LC combined with MS/MS: LC-MS/MS), the methods based on the use of arrays (RPPA) [7].

MS-based proteomic platforms for cancer studies and their principles of use are discussed in detail in [11]. To these platforms belong such methods as gel electrophoresis (1D-PAGE, 2D-PAGE (SDS-PAGE), 2D-DIGE), liquid chromatography (LC/MALDI or LC/MS (LC-MS/MS)), 2D-LC or multidimensional protein identification technology (MudPIT), LC-ESI-MS, mass spectrometry (ion sources (ESI MS, MALDI MS, SELDI MS) combined with mass analyzers (Q MS, TOF MS, FT-ICR MS): MALDI-TOF MS, SELDI-TOF MS, ESI-MS/MS). By the data [11], LC-MS/MS is used mostly with bottom-up strategy, along with this some methodologies based on top-down strategy are already developed, too [12]. Also, for identification of new cancer biomarkers and potential therapeutic targets LC-MS/MS could be combined with quantitative methods: ICAT-LC-MS/MS, ITRAQ-LC-MS/MS, SILAC-LC-MS/MS [11].

In general, modern proteomic studies often use gel electrophoresis and chromatography combined with MS. Mostly, gel electrophoresis and chromatography are used for separation of protein mixture into specific fractions containing few proteins with similar physical and chemical characteristics. The fractions could be further analyzed by MS, allowing identification of thousands of proteins per sample. During MS double scanning is used when information obtained after first scanning is selectively used during the second scanning. Apart from this, complex methods based on combination of few sequential separations of the proteins with the use of elementary LC methods (for example SCX-RP-LC [13], SCX-SCX-LC [14] or RP-RP-LC [15]) and their identification with the sequential use of elementary MS methods (for example, LC-MS/MS [16-19]) have been applied.

Analysis of the use of strategies and technologies. An analysis of proteomic studies of BC shows several major directions in this field.

Firstly, it is the development of the strategies of preparation of protein/peptide samples top-down or bottom-up with or without their proteolytic digestion (for example, trypsinization/pepsinization) prior to the use of proteomic technologies. An analysis of experimental studies of BC proteome at tissue level published in 2011-2016 has revealed that bottom-up strategy combined with modern technologies has been used more often (Table 1) due to its higher informativenes for identification of BC biomarkers [20]: on the one side, there has been revealed a trend for the use of unified bottom-up strategy, on the other side, for the use of both bottom-up and top-down strategies in one research but for different technological approaches. Also, there has been found a trend for minimal number of studies where top-down strategy was used along with narrow spectrum of methods (see Table 1). The authors [9] have listed wider spectrum of methods with which top-down strategy could be used for protein identification, but by our consideration, methodological variability of top-down in proteomic $B C$ research is some what depleted (see Table 1). This fact opens possibilities for analyzing the limitations of methods and equipment to overcome them for the proper use of top-down strategy for the analysis of complex protein mixtures and for the development of the optimal protocols on the use of this strategy with other methods and/or new technical solutions. An example of such optimization for the use of top-down is the work [20], reporting on successful usage of 2D-LC-MS/MS for identification of BC proteins in tumor tissue (see Table 1).

The second direction is the development of technologies via combination of several methods (chromatography and tandem MS). An analysis of experimental studies on BC proteomics from the point of applied technologies has revealed a trend for the widest use of combined LC-MS/MS with different modifications (see Table 1), where among elementary methods most commonly RP-LC, ESI MS (ion source) and LTQ-Orbitrap MS (tandem hybrid mass analyzer) were being used. For analysis of BC proteome triple mass analyzer XCT II MS (Triple Q MS) is used as well [21] (see Table 1). In the proteomic studies of different biologic material there appears a trend for the combined use of different elementary LC methods (SCX-RP-LC [13]), while in the studies of total BC proteome such trend is not observed - similar LC methods are used in tandem (SCX-SCX-LC [14] and RP-RP-LC [15]) (see Table 1). There is also a trend for prevalent use of label-free combined LC-MS/MS methods (label-free analysis, Table 1), that opens the possibilities for active use of methods utilizing affine labels (label analysis, Table 1) to achieve better quality of the results. In general, in proteomic studies employing MS, a wide spectrum of ion sources and mass analyzers has been used combination of which depended on the aim of the research. The use of one or another elementary methods was analyzed only for the studies where the methodological components were described in detail (see Table 1).

It is necessary to note that there have been reported methodologies, in particular, combination of both strategies to obtain maximally informative protein profile of the tumors [20], modification of the stage of peptide preparation for LC-MS/MS with analytical instruments in silico [19], development of new proteomic approach on the use of affine chromatography with top-down 
Table 1. Methods for modern proteomic strategies applied in the proteome research of $\mathrm{BC}$

\begin{tabular}{cll}
\hline \multicolumn{1}{c}{ Bottom-up } & \multicolumn{1}{c}{ Top-down } & \multicolumn{1}{c}{$\begin{array}{c}\text { Bottom-up + } \\
\text { top-down }\end{array}$} \\
\hline $\begin{array}{c}\text { Label-free analysis } \\
\text { LC-MS/MS: }\end{array}$ & $\begin{array}{l}\text { 1D-PAGE } \\
\text { (SDS-PAGE) [18], }\end{array}$ & $\begin{array}{l}\text { Label-free } \\
\text { analysis }\end{array}$ \\
LC-MS/MS [16, 17, 21, 25, 29, 2D-PAGE & 2D-LC-MS/MS \\
33, 36, 43-45, 79], (SDS-PAGE) & {$[20] ;$} \\
LC-MS/MS with isotope [14, 22, 28, 37, 46], & \\
dilution [23], 2D-DIGE [14, 36], & basic methods \\
nLC-MS/MS [18, 28, 41], affinity chroma- & of 2D-LC-MS/MS: \\
2D-LC-MS/MS [15, 22]; tography [22] & Bottom-up \\
basic methods of liquid & Proteomics: \\
chromatography for LC-MS/MS: & Nanospray MS, \\
RP-LC [21, 29, 36], & TripleTOF MS \\
2D-LC (RP-RP-LC) [15]; & [20]; \\
basic methods of mass spectrom- & Top-Down \\
etry for LC-MS/MS (ion sources): & Proteomics: \\
ESI MS [21, 22, 29, 25], & RP-LC, \\
SELDI MS [44]; & Nanospray MS, \\
basic methods of mass & Orbitrap MS \\
spectrometry for LC-MS/MS & [20] \\
(mass analyzers): &
\end{tabular}

Q MS [17], Q-TOF MS [28], LTQ MS [16, 45, 79], hybrid Q-Orbitrap MS $[33,43]$, LTQ-Orbitrap MS [15, 18, 22, 29 , $33,36,41]$,

XCT II MS (TripleQ MS) [21] reaction monitoring mass spectrometry:

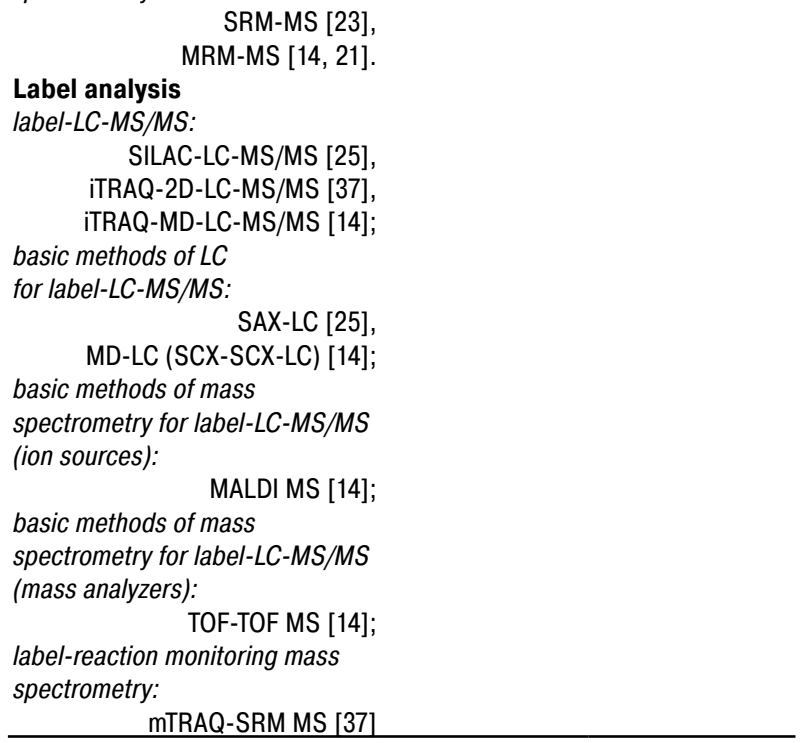

strategy [22], development of protocol for the use of LC-MS/MS with isotope dilution [23]. One should believe that along with the development of new proteomic analytical technologies for protein identification and achievement of the data on protein post-translational modifications there will be a drastic increase of the number of identified cancer biomarkers, including biomarkers of $\mathrm{BC}$ progression. For their further implication into clinical practice, an additional validation of identified proteins using the antibody-based methods is required, that, in turn, will stimulate the study of properties and functions of these proteins.

At present time proteomic-based search of $\mathrm{BC}$ biomarkers has a number of limitations at different levels:

- Biologic material. Molecular heterogeneity of BC, complex composition of biologic fluids used as ex- perimental samples, multiplicity of proteome composition and its dynamical variability create significant methodological challenge in proteomic research [24].

- Isolation, storage and preparation of experimental samples. Requirements for conditions of sample collection, their primary treatment, high quality storage conditions of biologic materials are being solved by standardization that is determined in part by special conditions preventing degradation of the particular proteins. There are some achievements in the standardization of plasma samples collection for obtaining plasma proteomic profile [21].

Disadvantages of bottom-up and top-down strategies could be referred to such limitations as well. Presently they are counter-balanced by technical solution for combination of the strategies in a joint method for proteomic profiling [20]. Apart from this, for tumor peptidome analysis an improved protocol of peptide preparation has been proposed that, being combined with the methods in silico, completes the results of bottom-up strategy [19].

There are still none technical means allowing simultaneous isolation and analysis of DNA, RNA and proteins from cryomaterials [24].

- Devices and instruments. In the samples low quantities of proteins that hypothetically could be tumor-specific, require perfect analytical sensitivity of the equipment. The methods of gelelectrophoresis are of the lowest sensitivity while the MS-based methods are of the highest sensitivity. MS possess own limitations as well at the levels of ion sources and mass analyzers, making impact into general disadvantages of combined elementary methods (for example, if ion sources MALDI MS or SELDI MS are combined with analyzers TOF MS - MALDI-TOF MS, SELDI-TOF MS [4]).

- Limitations in silico. The software (SW) used for assessment of experimental data at the stages of their analysis, visualization, storage, and interpretation should be up-graded or developed de novo [24]. Presently SW is used more and more often for verification and validation of the data [19, 20, 25]. The progress in silico will allow reaching higher levels of BC research [24], meta-analysis [24] and assessment of the obtained data.

Objects of study. The wide spectrum of human biologic material used for proteomic study of $\mathrm{BC}$, allows one to perform systemic analysis of tumor-host interactions. For sampling tumor tissue, invasive methods are used. Body fluids could be taken by noninvasive methods that is much more preferable for clinical application of experimental results.

In proteomic study of BC, a large number of monotypic samples are being used. Protein fractions are isolated from tumor or normal cells, tissues and body fluids [5, 7, 11, 26-33]. Biopsy, postoperative material, tissue obtained by laser capture microdissection (LCM) method [34, 35], BC cell lines in vitro, experimental tumors in vivo are studied in tumor tissue 
proteomics. In the research of body fluids, postoperative serum, tumor extracellular fluid, blood serum and blood plasma, mononuclear cells, cerebrospinal fluid, urine, saliva, milk, nipple aspirate, fluids from organs and body cavities are used. A typical scheme of modern proteomic studies of $B C$ is reviewed in details in [7], and illustrated in Fig. 4.

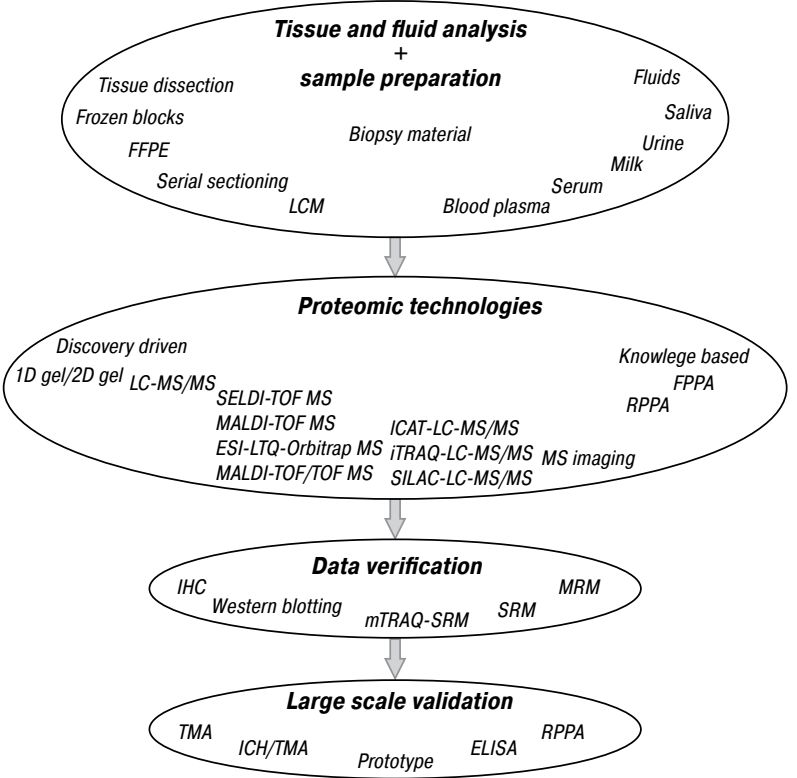

Fig. 4. Schematic representation of proteomic BC studies

Clinical importance of the results of proteomic studies of BC. Proteomic profiling of biologic material from BC patients with the use of MS-based methods allows detecting simultaneously much more individual proteins than antibody-based methods (including $\mathrm{IHC}$ [33]) applied for verification and validation of the results. Information obtained from proteomic analysis is useful for studying the role of extracellular matrix [15]; post-translational modification of proteins [31, 36]; proteins involved in DNA repair [33]; tumor micronvironment [33]; microenvironment of tumor cell [22]; proteins of tumor stroma [37, 38]; cytoplasmic proteins [39]; proteins of endoplasmic reticulum (EPR) [40]; the role of proteins of mechanistic pathways, components of protein biosynthesis, cyclins in progression, invasion and metastasis of $\mathrm{BC}$ [38].

The proteomic profiling based search for $\mathrm{BC}$ biomarkers showed following trends: BC progression (BC with different lymph nods status [37, 41, 42] and metastatic $B C[29,31,35])$, profiling of $B C$ subtypes (triple negative breast cancer (TNBC) [25, 37, 38, 41, 43, 44], HER2+ [14, 25, 37, 38, 44, 45], ER/PR [25, $37,38]$, basal and luminal $[19,20,45])$ and the study of tumors of different histopathological grade [22, 27].

In [46], the top-down strategy for 2D-PAGE (SDS-PAGE) and Bradford technique have been used for determination of expression levels of proteins in tumor and normal tissues of mammary gland. In total, 454 proteins have been found, 138 of which showed an altered expression in tumor tissue (expression of 61 proteins was suppressed, 3 - up-regulated, and 74-down-regulated). So, compared to normal tissue, expression of a large number of proteins is changed, and many of them are down-regulated, sometimes completely suppressed [46]. In our view, these data should have been verified by MS and LC-MS, because of low separating capacity of 2D-PAGE (SDS-PAGE).

Generation of large databases for proteomic profiles of biologic materials has limitations caused by variability of both the sample collection and proteomic technologies used for analysis. In [21], the collection of plasma samples from healthy individuals and $\mathrm{BC}$ patients has been standardized that allowed one to create database of proteomic profiles of plasma with the use of bottom-up strategy and combined LC-MS/MS method. This database will be useful for the search of BC biomarkers for diagnostic, prognosis, monitoring of the disease progression and therapy [21]. The data on three proteins - potential $\mathrm{BC}$ biomarkers, have been already verified (Table 2 ).

The recent findings in BC proteomic analysis pertaining to screening, diagnostics, therapy and prognosis are reviewed in detail in separate sections presented below.

Screening. Screening tests require high sensitivity, specificity, accuracy, non-invasiveness, ease of process, low cost and reliability of false-positive/ false-negative result, therefore proteomic analysis of biologic body fluids for identification of markers for preclinical changes could be the best choice for screening purposes.

Using combined LC-MS/MS and bottom-up strategy, protein biomarkers were identified in urine of $\mathrm{BC}$ patients with different disease stage and tumor material was studied in parallel as well [29]. Expression levels of 59 proteins was found to be different from that in control samples, in particular, 13 novel up-regulated proteins associated with $B C$ of diagnostic value have been revealed. The relation between $\mathrm{BC}$ progression and a panel of specific protein markers has been ascertained: preinvasive ductal carcinoma in-situ - leucine LRC36, protein MAST4 and uncharacterized protein $\mathrm{Cl} 131$, early invasive $\mathrm{BC}-$ DYH8, HBA, PEPA, MMRN2 proteins, filaggrin, and uncharacterized protein C4orf14 (CD014), and metastatic BC - AGRIN, NEGR1, FIBA proteins and KIC10 keratin. The proteins that have been already validated are listed in Table 2. These data will be used for the development of screening programs.

Diagnostics. Early diagnosis and monitoring of $\mathrm{BC}$ progression are of great importance for better prognosis of the disease.

TNBC is a heterogeneous pathology with unfavorable prognosis due to insufficient targeted treatment effectiveness. For the first time proteomic analysis of 12000 proteins and molecular profile of this BC subtype in tumor samples and cell cultures in vitro was provided using combined LC-MS/MS and bottomup strategy [43]. In this research proteins of signal pathways were quantified and proteins markers of drug resistance were identified. These data could be useful for understanding the mechanisms of drug resistance, as well as for diagnosis and therapy of TNBC. 
Table 2. The results of modern proteomic studies of BC

\begin{tabular}{llll}
\hline & Biological samples & \multicolumn{1}{c}{ Research methods } & \multicolumn{1}{c}{ Methods of valida } \\
\hline $\begin{array}{l}\text { Invasive } \\
\text { object }\end{array}$ & Tumor tissue of invasive duc- & 2D-PAGE (SDS-PAGE), & Western blotting \\
& tal carcinoma & 2D-DIGE, & MRM-MS \\
& Subtypes: & iTRAQ-MD-LC-MS/MS & \\
& Luminal B HER2+ve & (MD-LC (SCX-LC), & \\
& & \\
& & \\
& & \\
MALDI-TOF/TOF MS) & \\
& & \\
& & \\
& & \\
Tumor tissue of invasive duc- & 2D-PAGE (SDS-PAGE), & Western blotting \\
tal carcinoma & 2D-DIGE, & MRM-MS \\
Stages: & iTRAQ-MD-LC-MS/MS & \\
Early stages & (MD-LC (SCX-LC), & \\
& MALDI-TOF/OFF MS)
\end{tabular}

Late stages

Lymph node positive vs. negative, low grade primary BC tissues

Primary breast carcinoma tis- TRAQ-2D-LC-MS/MS sues from patients with different lymph node status

Breast ductal carcinoma tis- Published data and data- IHC/TMA sues base (mRNA level)

Metastatic BC (tumor tissue) Published data and data- IHC/TMA base (mRNA level)

Breast tumor tissues HER2+ LC-MS/MS (SELDI MS) IHC TNBC

Human disease-free breast LC-MS/MS with isotope SRM-MS tissues and malignant breast dilution tumors

TC/TMA:
$B C$ tissues with different $E R$, Published data on proPR and HER2 status (meta- teins as important taranalysis) gets and proteomic processes in BC

RPPA

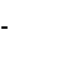

(1)

\footnotetext{
Non-in- Serum (patients with recurrent Lectin affinity chroma-

vasive $\mathrm{BC}$ and patients with no sign tography, 2D-DIGE,

object of recurrence 5 years after di- LC-MS/MS (RP-LC) agnosis)
Plasma (healthy donors and LC-MS/MS
BC patients) (RP-LC, ESI MS, XCT II MS (TripleQ MS)

Com- Urine and tumor tissue (iden- LC-MS/MS (RP-LC)

bined tification)

object Cell lines (validation)

Tumor tissue (validation)
}

ELISA
MRM-MS

PCR (transcript level), Transgelin (TAGLN)

TRAQ-2D-LC-MS/MS

mTRAQ-SRM MS,

mTRAQ-SRM MS, Transgelin (TAGLN);

Transgelin-2 (TAGLN2)

Apolipoprotein A1 (APOA1)

Protein biomarkers of $B C$

Tumor subtyping, diagnosis

Heat shock protein HSP 90-beta

(hs90b);

Eukaryotic elongation factor

of early and late stages

1 alpha (EF1A1); Peroxiredoxin

3 (PRDX3); NHRF1.

Peroxiredoxin 1 (PRDX1); Oxidoreductase (catD); Calreticulin (CALR)

ATPase beta chain (atpB);

SOX14 (CH60) SRY-box 14.

Tropomyosin 4 (TPM4); Oxido-

reductase (catD); Peroxiredoxin

3 (PRDX3); Annexin A3 (ANXA3);

Heat shock protein family $B$ (small) member 1 (HSPB1).

Prediction of treatment outcome

Calreticulin (CALR); Ovotransferrinlike (TRFE); Gelsolin (GELS);

SOX14 (CH60) SRY-box 14;

Capping actin protein, gelsolin like (CAPG);

Ywhag (1433G) tyrosine 3-monooxygenase/tryptophan 5-monooxygenase activation protein gamma; Glucose regulated protein

78 (grp78);

NHRF1.

Protein biomarkers of $\mathrm{BC}$

Tumor subtyping,

diagnosis of early and late

stages

Prediction of treatment out-

come

Kinesin associated protein 3

(KIFAP3)

Ribosome binding protein

1 (RRBP1)

KRT19 (CK19) keratin 19.

Cancer-associated biomark-

ers of lymph node metasta-

RNA-binding Ras-GAP SH3 binding protein (G3BP)

Apurinic/apyrimidinic endonuclease 1(APE1)

ER; PR; Apoptosis regulator (BCL2); GATA binding protein 3 (GATA3);

KIAA1324 (EIG121); Epidermal growth factor receptor (EGFR); Erb-b2 receptor tyrosine kinase 2 (HER2); HER2p1248; Cyclin B1 (CCNB1); Cyclin E1 (CCNE1).

CDH5 (CADHERIN5) cadherin 5, Predictive and diagnostic biotype 2 (vascular endothelium) marker

Apolipoprotein A1 (APOA1); Hemopexin hemopexin-like; Angiotensin preprotein. Extracellular matrix protein 1 (ECM1);

Western blotting FLG2 (FILAGGRIN) filaggrin fami-

IHC, Western blotting ly member 2; Microtubule associated serine/threonine kinase family member 4 (MAST4);

Microtubule associated serine/ threonine kinase family member 4 (MAST4). 
Variability of HER2/Neu overexpression is typical for molecular subtypes of invasive ductal carcinoma, luminal B HER2+ (ER+/PR+/HER2+) and HER2 enriched (ER-/PR-/HER2+), which are poorly studied yet in regard to prognostic markers. A comparative proteomic profiling of luminal B HER2+ve and HER2 enriched subtypes of invasive ductal carcinoma and healthy tissues of mammary gland was provided [14]. Tumor material obtained during modified radical mastectomy has been used for the search of protein biomarkers of early and late stages of these molecular BC subtypes with the use of proteomic analysis methods (see Table 2). Top-down strategy was used for gel-electrophoresis, and bottomup strategy - for MS-based methods. In total, in the studied BC subtypes 67 proteins expressed in tumor material were found, and expression of 68 proteins depended on BC stages; there have been validated (see Table 2) 6 proteins for luminal B HER2+ subtype, 5 proteins for HER $2+$ subtype, 5 and 8 proteins for early and late stages of these $B C$ subtypes, respectively. The authors believe that these panels of protein biomarkers could be used for molecular classification of the tumors in diagnostics of early and late BC stages and for prognosis of treatment outcome.

Several studies analyzed expression of protein isoforms and proteins which composition and functions were altered via post-translational modifications (phosphorylation, acetylation, glycosylation, methylation and ubiquitination) [11, 31, 36]. The results obtained are proposed for the use as sensitive diagnostic markers of BC clinical course. In studies mentioned above, the samples of blood serum and urine of $\mathrm{BC}$ patients and paraffin blocks of primary tumors (FFPE), were analyzed by lectin microarray [31] or gel-electrophoresis as top-down strategy combined with MS-based methods as bottom-up strategy [36] (see Table 2). As it has been concluded [31, 36], the altered glycosylation of proteins in cancer patients could be associated with particular cancer types, however, total spectrum of glycane structures is still unknown. An analysis of glycosylated proteins of blood serum and urine of patients with metastatic $B C$ has revealed diagnostic and predictive potential of cadherin-5 and lectin-binding patterns, including $\mathrm{N}$ - and O-bound glycanes [31, 36]. This is supported by validation of the results [36] establishing $90 \%$ specificity of cadherin- 5 as diagnostic marker of metastasis (see Table 2).

Therapy. Proteomic profiling of BC specimens could be also useful for analyzing mechanism of action of anticancer agents such as identification of targets [25, 44], search for protein-targets or their inhibitors for adjuvant chemotherapy [23, 41] and controlling invasive properties of tumors via influence on proteins of the cells surrounding tumors [33].

In regard to the recent results of integrated "genome-transcriptome" studies in the absence of universal panel of $\mathrm{BC}$ biomarkers and optimal medicinal remedies the proteomic analysis of tumor tissues of different BC subtypes is of special importance.
Using bottom-up strategy, quantification technology SILAC-LC-MS/MS and LC-MS/MS on FFPE BC tissue blocks (ER+/PR+, HER2+, TNBC) and BC cell lines (HCC1599, MCF7, HCC1937) the study of functional networks between multifunctional proteins and cell processes in the tumors of different molecular subtypes has been conducted [25]. Up to 410,000 proteins have been analyzed, and it has been shown that $B C$ subtypes differ in the functions of proteins involved in translation of mRNA, cell growth, intercellular interaction, and energetic metabolism. In total, 19 protein signatures were found, just 3 from which were related to gene copy number, and 11 - to mRNA levels. Possibly, these data could support an absence of regular relations between the protein product level and gene copy number, and protein product content and mRNA profile. The special SW was applied for a cross-validation procedure of the obtained data on proteomic profiling of the tumors. These results embody the novel ideas that are practically valid for the development of specific therapeutic agents.

Predictive protein markers of different BC subtypes will allow us to determine therapeutic response to particular treatment, to optimize and personalize cancer therapy.

In a pilot study [44], protein signatures of two BC subtypes potentially useful for prediction of treatment results were identified. Specific predictive protein markers of response to neoadjuvant chemotherapy were studied using bottom-up strategy and combined LC-MS/MS method in tumors of HER2+ and TNBC subtypes. There were identified 20 protein signatures typical for tumors of both subtypes, 20 signatures with different expression levels allowing to classify these subtypes, 20 predictive markers of response to neoadjuvant chemotherapy for HER2+ subtype and 30 predictive markers of response to neoadjuvant chemotherapy for TNBC subtype. TNBC subtype was characterized by overexpression of ALDH1A1 and galectin-3-binding protein, while in HER2+ subtype the following proteins were found to be overexpressed: transketolase, transferrin, CK19, thymosin $\beta 4$, and thy$\operatorname{mosin} \beta 10$. The number of proteins, namely, enolase, peroxiredoxin 5, periostin precursor, cathepsin D preproprotein, vimentin, Hsp 70, annexin 1, RhoA were related to the tumor response to neoadjuvant chemotherapy. Also, two proteins for classification of these subtypes were validated (see Table 2).

In spite of constantly increasing number of clinical trials of anticancer agents there is a necessity for the correction of modern treatment schemes from the point of benefit/risk ratio. As far as TNBC is highly aggressive and there are still none sensitive specific prognostic markers, up-to-date an optimal target therapy of this subtype isn't developed. As a rule, the patients with negative lymph node status are cured with adjuvant chemotherapy, but in $30 \%$ of cases distant metastasis develops $[47,48]$. With the use of bottomup strategy and technology nLC-MS/MS [41] in tumor material of patients with TNBC and negative lymph 
node status not treated with adjuvant chemotherapy, 11 prognostic protein signatures, protein products of the CMPK1, AIFM1, FTH1, MTHFD1, EML4, GANAB, CTNNA1, AP1G1, STX12, AP1M1, CAPZB genes were identified and verified. The obtained results could be useful in clinical practice and address an expediency of adjuvant systemic therapy in patients with TNBC and negative lymph node status.

The search for a candidate for proteomic biomarker for prognosis and therapy of BC patients has been recently attempted [23]. Overexpression of APE13 (the main protein of DNA excision repair pathway apurinic/ apyrimidinic endonuclease) was detected in clinical material with the use of bottom-up strategy and the developed analytical approach based on MS [23] (see Table 2). Hyper-/hypoexpression of APE1 could be possibly related to decreased/increased tumor cell survival rate, therefore in future its inhibitors could be used in clinical practice [23]. It is supposed that APE1 expression levels could be related to life expectancy of $B C$ patients, and clinical assessment of APE1 expression levels in intact and tumor tissues of mammary gland could be of prognostic and predictive value [23].

Cancer-associated fibroblasts (CAFs) are known to stimulate angiogenesis and metastasis $[49,50]$ and an inflammatory and wound healing related activation of fibroblasts are the main mechanisms of CAF activation [33]. The functional state of CAFs was assessed in biopsy specimens of breast adenocarcinoma using combined method LC-MS/MS and bottom-up strategy [33]. In total, 2074 proteins from biopsy material fibroblasts and 5212 proteins from cultured ZR-75-1 cells were identified. Comparative analysis of proteins of untreated fibroblasts, fibroblasts incubated with IL-1 $\beta$ (in vitro modeling of inflammatory way of fibroblast activation) or TGF- $\beta$ (in vitro modeling of wound healing-induced activation of fibroblasts) has shown that proteomic profile of BC biopsy could be useful for assessment of cell types at quiescent state, inflammation, wound healing. Proteomic profile of CAFs was found to be close to that of fibroblasts at the state of wound healing (common proteins, including fibulin-5, SLC2A1 and MUC18). The authors supposed [33] model CAFs systems could be advantageous for testing the agents which inhibit or reverse the proinvasive activity of the components of tumor microenvironment.

Prognosis. At present time the existing clinical criteria of pathologic process based on tumor aggressiveness grading don't reflect a real state of cancer process for assessment of its progression and prognosis [22]. With the use of top-down strategy (methods: affinity chromatography, 2D-PAGE (SDS-PAGE)) and bottom-up strategy (combined method LC-MS/MS) [22] protein signatures associated with histopathological grading (G1, G2, G3) of breast tumors were indentified, 49 of which were validated using the data of meta-analysis of transcription profiling of tumors of independent group of patients. The special SW permitted to determine that the validated proteins are localized in intercellular space, plasma membrane, cytoplasm, and nuclei. The obtained results could be important for the revision of the microenvironment model during tumor progression and be useful for classification and prognosis of BC.

Two proteins, transgelin and transgelin-2 could be of clinical importance serving as prognostic proteomic markers of metastasis of different tumor types (pancreatic, colorectal, gastric, lung, BC) [37, 51-54]. Transgelin is a differentiation marker of smooth muscles [55], and is expressed in myofibroblasts and CAFs of gastric and lung tumors $[54,56]$. Its up-regulation in fibroblasts in gastric tumor tissue supports tumor cell migration and invasion via increased production of matrix metalloproteinase-2 [52], and this protein is oncosuppressor, expression of which is downregulated by Ras oncoprotein in BC samples [57]. Hypermethylatioin of its promoter is related to downregulation of its expression in cell lines and tumor tissues of mammary gland [58]. In regard to transgelin-2, its overexpression in breast tumor vasculature has been reported [59]. Using proteomics methods, a comparative analysis of expression of transgelin and transgelin-2 in lymph nodes of BC patients has been provided [37]. Using top-down strategy and 2D-PAGE (SDS-PAGE) up-regulation of transgelin in positive lymph nodes of BC patients with primary low grade tumors and different lymph node status has been revealed (see Table 2 ). These results were clinically validated on the larger group of BC patients with different lymph node status (see Table 2). In this research [37], a comparative proteomic analysis of transgelin and transgelin-2 in tumor tissue of BC patients with the use of bottom-up strategy demonstrated a specific relation between transgelin and lymph node metastasis in BC patients and tumor differentiation grade, nevertheless no association of transgelin expression with molecular markers ER, PR, HER2 has been found. Since both presence [60] and absence [37] of specific expression of transgelin have been reported, its specificity as a marker is under question. Down-regulation of transgelin in high grade tumors and overexpression of transgelin-2 in metastatic and low differentiated tumors were considered as a consequence of stromal cells dedifferentiation [37]. It has been shown (IHC/TMA) that transgelin is mostly expressed in stromal cells (fibroblasts and endothelial cells), while transgelin- 2 is expressed in epithelial cells of the tumors $[37,56]$. The authors $[37,56]$ supposed that tumor stroma is capable to express relevant proteomic biomarkers of potential clinical importance.

The studies on gene expression profiling have created large databases for genes, RNA and proteins expressed in BC. In particular, in tumor samples of $B C$ patients and cell lines [61] overexpression of KIFAP3 gene (located in 1q24 chromosome loci, and coding for kinesin associated protein 3 (KIFAP3) has been revealed [39]. Protein KIFAP3 is localized in nucleus, cytoplasm and EPR $[62,63]$ and interacts with the proteins involved in carcinogenesis: interaction of KIFAP3 with APC affects cell migration [64, 
65], KIFAP3 is phosphorylated with BRK or PTK6 kinases in BC cell line BT20 [66], KIFAP3 is required for BRK-induced cell migration [66] and may play a role of a key effector of BRK signal pathway [66]. Experimental study on validation of KIFAP3 protein [39] (see Table 2) has shown its overexpression in the cells of breast ductal carcinoma, mostly in cytoplasm. Expression of one more protein considered as BCassociated markers, namely RRBP1 (ribosome binding protein), was studied [40]. RRBP1 is a multifunctional membrane protein localized in rough EPR [67-69], cytoplasm and nucleus [70], participating in translocation of nascent proteins through the membrane of rough EPR [71]. RRBP1 interacts with KIF5B [72] and is involved in ribosome binding [71], biosynthesis of procollagen and terminal differentiation of secretory tissues $[67,73]$. High level of its expression was found in some cancer cell lines [74], and its overexpression was registered in colorectal cancer [75]. Overexpression of RRBP1 in the perinuclear region of cytoplasma was documented in $84 \%(177 / 219)$ cases of breast carcinoma [40] (see Table 2). These two examples demonstrate an integrated interaction between the data and sources of "omics" and systemic assessment of functions of BC-associated proteins.

Systemic neoadjuvant therapy may increase the risk of recurrence after organ-sparing operations and promote the development of drug resistance [76-78]. Modern functional proteomics could be helpful for prognosis of pathologic response to systemic neoadjuvant therapy. Proteomic meta-analysis utilizing RPPA method covering tumor specimens from $712 \mathrm{BC}$ patients who received taxane and anthracycline-taxane systemic therapy, has validated a panel from 10 predictive biomarkers (see Table 2) [38]. Based on these findings, the patients may be stratified into 6 prognostic groups: HER2+; ER-/PR- and ER-/PR-/HER2 with unfavorable prognosis; ER+/PR+ with favorable prognosis; and three intermediate groups that mostly were characterized by overexpression of tumor cell proteins involved in various cell processes (cyclines, components of protein biosynthesis system, stromal markers, proteins of mechanistic pathways).

For better stratification of the patients at the stage of prescription of adjuvant chemotherapy and for prognosis of the disease course in the study [42] the blood serum samples of patients with primary BC and lymph node metastase have been analyzed postoperatively. With the use of ion-exchange and affine chromatography (immobilized metal affinity chromatography IMAC) combined with SELDI-TOF MS protein profiling and MALDI-TOF/TOF MS for peptide profiling 4 mass peaks were revealed $(\mathrm{m} / \mathrm{z} 3073, \mathrm{~m} / \mathrm{z} 3274$, $\mathrm{m} / \mathrm{z} 4405$ and $\mathrm{m} / \mathrm{z}$ 7973) believed typical of proteins associated with recurrence-free survival of the patients. Among these potential biomarkers, a protein with $\mathrm{m} / \mathrm{z} 3274$ was identified as an inter-alpha-trypsin inhibitor heavy chain 4 fragment. These data should be further validated with enrollment of an independent group of patients, however, the authors consider the use of anion-exchange fractionation combined with SELDI-TOF MS as a promising tool for identification of new prognostic markers of BC [42].

Improvement of quality of prteomic BC studies. The studies of BC proteome and peptidome aimed at the search of diagnostic and prognostic markers develop dynamically, especially in regard to validated clinical results. Such aim requires perfection of methodological and technical approaches for the analysis and identification of various BC biomarkers. As an example of the newest approaches one could mention combining existing strategies and developing new analytical platforms.

Combined strategies. A large number of works reviewed in [9] addressed advantages and disadvantages of bottom-up and top-down strategies for different tasks, and also expediency of their use for proteomic analysis and quantification of protein molecules.

In the study [20], the complementarity of these strategies was assessed with the use of combined method LC-MS/MS and material of two BC models, namely, patient-derived xenografts established from a basal-like and luminal B BC subtypes. The study has been designed as follows: testing of label-free top-down quantitative proteomics platform (as far as LC-MS/MS is used mostly with bottom-up strategy); comparative analysis of differential expression of proteins and their proteoforms with low molecular weight $(<30 \mathrm{kDa})$ in the samples of basal and luminal $\mathrm{B}$ molecular BC subtypes. The comparative analysis of the efficacy of using bottom-up and top-down strategies supported the 10 -fold superiority of bottomup: identification of 49,185 groups of peptides and quantification of 3519 proteins derived from them versus 982 proteoforms and 358 proteins in the case of top-down use. However, quantitative effectiveness of the strategies had a ratio of 60:40, and the use of top-down allowed to gain a unique information complementing the data obtained with the use of bottom-up. In turn, bottom-up was by 8 times more accurate for identification of proteins with molecular weight of $0-30 \mathrm{kDa}$. With the use of special SW, the obtained data were validated. This work demonstrated the priority of combination of these strategies in the study of $\mathrm{BC}$ proteome and $\mathrm{BC}$ biology involving genome data, and also confirmed that bottom-up strategy does not allow identifying the differences between some post-translational modifications (for example, phosphorylation).

New analytic platform. Tumor peptidome (intracellular and intercellular products of protein degradation) could represent a potential source of biomarkers for tumor-related proteolytic properties. Using combined method LC-MS/MS, an analytic platform in silico has been developed which along with improved protocol of peptides isolation complemented the results of conventional bottom-up strategy. This platform has been used for a complex analysis of peptidome of ovarian cancer and xenografts of basal and luminal BC subtypes [19]. The developed platform 
represents the novel technological stage for further determination of molecular features and functional significance of peptidomic/degradomic activities in tumor tissues. It is characterized by reproducibility of the results and high capacity of studies on quantification of identified peptides. The use of the platform allowed one to identify peptidome profiles reflecting the types of action of tumor-associated proteases; the results were validated with the use of special SW. The developed analytic platform and the obtained data are of practical significance not only for tumor tissue profiling in $\mathrm{BC}$, but also in other cancer types as far as aberrant degradation of proteins is inherent to many of tumor types.

Methods for verification and validation of the results of proteomic analysis. Verification and validation of the results of experimental studies are the mandatory stages is analyzing the bulk of findings based on the generally accepted methods. Validation means that from the pool of identified proteins only relevant oncological markers of clinical significance should be selected. Three groups of methods each with its advantages and disadvantages, are being used for this purpose: IHC based, TMA based, and MS based (SRM/MRM-MS) [7]. Combined IHC/TMA group of validation methods is becoming more and more popular $[37,39,40]$, because it allows to analyze the samples of tissues of larger size compared to convenient TMA [7]. Common methods are Western blot [14, 29] and MRM-MS [14, 21] while SRM-MS [23], MTRAQ-SRM MS [37], IHC [44], RPPA [38] and ELISA are less commonly used [36]. There is a trend for the use of several validation methods in one study for more effective assessment of the significance of the obtained results (Western blot + MRM-MS [14], mTRAQ-SRM MS + IHC/TMA [37], Western blot + IHC [29], Table 2). It's necessary to note that RPPA has been used as the validation method in proteomic meta-analysis of $\mathrm{BC}$ tissue $(n=712)$ aimed at determination of predictive biomarkers panel ([38], Table 2). The use of SW for verification and validation of experimental results becomes more common $[19,20,25]$. The results of proteomic studies which were successfully validated are used as clinically valid biomarkers for diagnostics, prognosis and therapy of BC [29, 36-40,44].

In conclusion, an analysis of literature sources on $\mathrm{BC}$ proteomics indicated an important role of topdown and bottom-up strategies as the major ones in the search of proteomic BC biomarkers with the use of LC-MS/MS. The technological progress is focused on more wide use of a spectrum of elementary LC and MS methods in a frame of combined LC-MS/MS. The study of BC proteome is directed on profiling of various biologic materials and is aimed at the improvement of prophylaxis, screening, diagnostics, prognosis, and therapy. A large pool of proteins of mammary gland tumors and BC-associated proteins from body fluids have been already identified, and in part they were validated. The progress of validation methods is helpful in more efficient application of BC biomarkers in clinical practice. Taken together, the results of proteomics studies demonstrate an integrated interaction of the data and "omics" sources with the systemic approach for assessment of functions of biomolecules in various pathologies and $\mathrm{BC}$ in particular.

\section{REFERENCES}

1. Chekhun VF. From systemic biology of cancer to the methodology of personalized therapy. Oncologiya 2012; 14: 84-8 (In Russian).

2. Maydannik VG. Global trends in the development of scientific research in clinical medicine. Zh Nat Acad Med Sci Ukr 2014; 20: 416-25 (In Ukrainian).

3. Khaytovich MV. Personalized medicine: modern state and perspectives. Ukr Sci-Med Youth Zh № 2 2015; 88: 6-11 (In Ukrainian).

4. Gholizadeh M, Parizadeh SA, Pasandideh R. Proteomics and bioinformatics approaches for breast cancer researches. Int J Agri Crop Sci 2013; 5: 1863-8.

5. Drake RR, Cazares LH, Jones E, et al. Challenges to developing proteomic-based breast cancer diagnostics. OMICS 2011; 15: 251-9.

6. Qin XJ, Ling BX. Proteomic studies in breast cancer (review). Oncol Lett 2012; 3: 735-43.

7. Gromov P, Moreira JMA, Gromova I. Proteomic analysis of tissue samples in translational breast cancer research. Expert Rev Proteomics 2014; 11: 285-302.

8. Kulasingam V, Diamandis EP. Strategies for discovering novel cancer biomarkers through utilization of emerging technologies. Natl Clin Pract Oncol 2008; 5: 588-99.

9. Yates JR, Ruse CI, Nakorchevsky A. Proteomics by mass spectrometry: approaches, advances, and applications. Ann Rev Biomed Eng 2009; 11: 49-79.

10. Chae YK, Gonzalez-Angulo AM. Implications of functional proteomics in breast cancer. Oncologist 2014; 19: 328-35.

11. Khadir A, Tiss A. Proteomics approaches towards early detection and diagnosis of cancer. J Carcinog Mutagen 2013; S14: 002.

12. Ntai I, Kim K, Fellers RT, et al. Applying label-free quantitation to top down proteomics. Anal Chem 2014; 86: 4961-8.

13. Yang F, Shen Y, Camp DG, et al. High-pH reversedphase chromatography with fraction concatenation for 2D proteomic analysis. Expert Rev Proteomics 2012; 9: 129-34.

14. Pendharkar N, Gajbhiye A, Taunk K, et al. Quantitative tissue proteomic investigation of invasive ductal carcinoma of breast with luminal B HER2 positive and HER2 enriched subtypes towards potential diagnostic and therapeutic biomarkers. J Proteomics 2016; 132: 112-30.

15. Maller O, Hansen KC, Lyons TR, et al. Collagen architecture in pregnancy-induced protection from breast cancer. J Cell Sci 2013; 126: 4108-10.

16. Zhang F, Wang M, Michael T, et al. Novel alternative splicing isoform biomarkers identification from high-throughput plasma proteomics profiling of breast cancer. BMC Syst Biol 2013; 7: S8.

17. Zhang F, Deng Y, Wan, M, et al. Pathway-based biomarkers for breast cancer in proteomics. Cancer Inform 2014; 13: 101-8.

18. Dowling $P$, Palmerini V, Henry M, et al. Transferrinbound proteins as potential biomarkers for advanced breast cancer patients. BBA Clinical 2014; 2: 24-30.

19. $\mathrm{Xu} \mathrm{Z,} \mathrm{Wu} \mathrm{C,} \mathrm{Xie} \mathrm{F,} \mathrm{et} \mathrm{al.} \mathrm{Comprehensive} \mathrm{quantita-}$ tive analysis of ovarian and breast cancer tumor peptidomes. J Proteome Res 2014; 14: 422-33.

20. Ntai I, LeDuc RD, Fellers RT, et al. Integrated bottom-up and top-down proteomics of patient-derived breast tumor xenografts. Mol Cell Proteomics 2016; 15: 45-56. 
21. Riley CP, Zhang X, Nakshatei H, et al. A large, consistent plasma proteomics data set from prospectively collected breast cancer patient and healthy volunteer samples. J Transl Med 2011; 9: 80.

22. Olsson N, Carlsson $P$, James $P$, et al. Grading breast cancer tissues using molecular portraits. Mol Cell Proteomics 2013; 12: 3612-23.

23. Coskun E, Jaruga P, Reddy PT, et al. Extreme expression of DNA repair protein apurinic/apyrimidinic endonuclease 1 (APE1) in human breast cancer as measured by liquid chromatography and isotope dilution tandem mass spectrometry. Biochemistry 2015; 54: 5787-90.

24. Goncalves A, Bertucci F. Clinical application of proteomics in breast cancer: state of the art and perspectives. Med Princ Prac 2011; 20: 4-18.

25. Tyanova $\mathrm{S}$, Albrechtsen $\mathrm{R}$, Kronqvist $\mathrm{P}$, et al. Proteomic maps of breast cancer subtypes. Nat Commun 2016; 7: 10259.

26. Morrison C, Mancini S, Cipollone J, et al. Microarray and proteomic analysis of breast cancer cell and osteoblast co-cultures. J Biol Chem 2011; 286: 34271-85.

27. Al-Tarawneh SK, Border MB, Dibble CF, et al. Defining salivary biomarkers using mass spectrometry-based proteomics: a systematic review. OMICS 2011; 15: 353-61.

28. Aslebagh R, Ngounou A, Channaveerappa D, et al. Proteomics study of human breast milk for breast cancer biomarkers discovery. FASEB J 2015; 29: 567.26.

29. Beretov J, Wasinger VC, Millar EK, et al. Proteomic analysis of urine to identify breast cancer biomarker candidates using a label-free LC-MS/MS approach. PLoS One 2015; 10: e0141876.

30. Di Luca A, Henry M, Meleady $P$, et al. Label-free LC-MS analysis of HER2+ breast cancer cell line response to HER2 inhibitor treatment. Daru 2015; 23: 40.

31. Fry SA, Afrough B, Lomax-Browne HJ, et al. Lectin microarray profiling of metastatic breast cancers. Glycobiology 2011; 21: 1060-70.

32. Gast MCW, Zapatka M, van Tinteren H, et al. Postoperative serum proteomic profiles may predict recurrence-free survival in high-risk primary breast cancer. J Cancer Res Clin Oncol 2011; 137: 1773-83.

33. Groessl M, Slany A, Bileck A, et al. Proteome profiling of breast cancer biopsies reveals a wound healing signature of cancer-associated fibroblasts. J Proteome Res 2014; 13: 4773-82.

34. Fuller AP, Palmer-Toy D, Erlander MG, et al. Laser capture microdissection and advanced molecular analysis of human breast cancer. J Mammary Gland Biol Neoplasia 2003; 8: 335-45.

35. Espina V, Wulfkuhle J, Liotta LA. Application of laser microdissection and reverse-phase protein microarrays to the molecular profiling of cancer signal pathway networks in the tissue microenvironment. Clin Lab Med 2009; 29: 1-13.

36. Fry SA, Sinclair J, Timms JF, et al. A targeted glycoproteomic approach identifies cadherin- 5 as a novel biomarker of metastatic breast cancer. Cancer Lett 2013; 328: 335-44.

37. Dvořáková M, Jeřábková J, Procházková I, et al. Transgelin is upregulated in stromal cells of lymph node positive breast cancer. J Proteomics 2016; 132: 103-11.

38. Gonzalez-Angulo AM, Hennessy BT, Meric-Bernstam F, et al. Functional proteomics can define prognosis and predict pathologic complete response in patients with breast cancer. Clin Proteomics 2011; 8: 11.

39. Telikicherla D, Maharudraiah J, Pawar H, et al. Overexpression of kinesin associated protein 3 (KIFAP3) in breast cancer. J Proteomics Bioinform 2012; 5: 122-6.

40. Telikicherla D, Marimuthu A, Kashyap MK, et al. Overexpression of ribosome binding protein 1 (RRBP1) in breast cancer. Clin Proteomics 2012; 9: 7.
41. Liu NQ, Stingl C, Look MP, et al. Comparative proteome analysis revealing an 11-protein signature for aggressive triple-negative breast cancer. J Natl Cancer Inst 2014; 106: djt376.

42. Gast MCW, Zapatka M, van Tinteren H, et al. Postoperative serum proteomic profiles may predict recurrence-free survival in high-risk primary breast cancer. J Cancer Res Clin Oncol 2011; 137: 1773-83.

43. Lawrence RT, Perez EM, Hernández D, et al. The proteomic landscape of triple-negative breast cancer. Cell Rep 2015; 11: 630-44.

44. He J, Whelan SA, Lu M, et al. Proteomic-based biosignatures in breast cancer classification and prediction of therapeutic response. Int J Proteomics 2011; 2011: 1-16.

45. Zhang F, Chen JY. Breast cancer subtyping from plasma proteins. BMC Med Genomics 2013; 6: S6.

46. Behboodi F, Tavirani MR, Yousefzadeh S, et al. Studying the proteomic pattern of cancerous tissue in patients with breast cancer and its' comparing with healthy breast. Zahedan J Res Med Sci 2015; 17: e2197.

47. Harris L, Fritsche H, Mennel R, et al. American Society of Clinical Oncology 2007 update of recommendations for the use of tumor markers in breast cancer. J Clin Oncol 2007; 25: 5287-312.

48. Dent R, Trudeau M, Pritchard KI, et al. Triple-negative breast cancer: clinical features and patterns of recurrence. Clin Cancer Res 2007; 13: 4429-34.

49. Mantovani A, Schioppa T, Porta Cet al. Role of tumorassociated macrophages in tumor progression and invasion. Cancer Metastasis Rev 2006, 25: 315-22.

50. Micke P, Östman A. Exploring the tumour environment: cancer-associated fibroblasts as targets in cancer therapy. Expert Opin Ther Targets 2005; 9: 1217-33.

51. Lin Y, Buckhaults PJ, Lee JR, et al. Association of the actin-binding protein transgelin with lymph node metastasis in human colorectal cancer. Neoplasia 2009; 11: 864-73.

52. Yu B, Chen X, Li J, et al. Stromal fibroblasts in the microenvironment of gastric carcinomas promote tumor metastasis via upregulating TAGLN expression. BMC Cell Biol 2013; 14: 17

53. Zhou L, Zhang $\mathrm{R}$, Zhang $\mathrm{L}$, et al. Up-regulation of transgelinis an independent factor predictive of poor prognosis in patients with advanced pancreatic cancer. Cancer Sci 2013; 104: 423-30.

54. Rho J-H, Roehrl MHA, Wang JY. Tissue proteomics reveals differential and compartment-specific expression of the homologs transgelin and transgelin-2 in lung adenocarcinoma and its stroma. J Proteome Res 2009; 8: 5610-8.

55. Duband JL, Gimona M, Scatena M, et al. Calponin and SM 22 as differentiation markers of smooth muscle: spatiotemporal distribution during avian embryonic development. Differ Res Biol Divers 1993; 55: 1-11.

56. Dvorakova M, Nenutil R, Bouchal P. Transgelins, cytoskeletal proteins implicated in different aspects of cancer development. Expert Rev Proteomics 2014; 11: 149-65.

57. Shields JM, Rogers-Graham K, Der CJ. Loss of transgelin in breast and colon tumors and in RIE-1 cells by Ras deregulation of gene expression through Raf-independent pathways. J Biol Chem 2002; 277: 9790-9.

58. Sayar N, Karahan G, Konu O, et al. Transgelin gene is frequently down-regulated by promoter DNA hypermethylation in breast cancer. Clin Epigenetics 2015; 7: 104.

59. Hill JJ, Tremblay T-L, Pen A, et al. Identification of vascular breast tumor markers by laser capture microdissection and label free LC-MS. J Proteome Res 2011; 10: 2479-93.

60. Rao D, Kimler BF, Nothnick WB, et al. Transgelin: a potentially useful diagnostic marker differentially 
expressed in triple-negative and nontriple-negative breast cancers. Hum Pathol 2015; 46: 876-83.

61. Grigoriadis A, Mackay A, Reis-Filho JS, et al. Establishment of the epithelial-specific transcriptome of normal and malignant human breast cells based on MPSS and array expression data. Breast Cancer Res 2006; 8: R56.

62. Shimizu K, Kawabe H, Minami S, et al. SMAP, an Smg GDS-associating protein having arm repeats and phosphorylated by Src tyrosine kinase. J Biol Chem 1996; 271: 27013-7.

63. Shimizu K, Shirataki H, Honda T, et al. Complex formation of SMAP/KAP3, a KIF3A/B ATPase motorassociated protein, with a human chromosome-associated polypeptide. J Biol Chem 1998; 273: 6591-4.

64. Jimbo T, Kawasaki Y, Koyama R, et al. Identification of a link between the tumour suppressor APC and the kinesin superfamily. Nat Cell Biol 2002; 4: 323-7.

65. Teng J, Rai T, Tanaka Y, et al. The KIF3 motor transports N-cadherin and organizes the developing neuroepithelium. Nat Cell Biol 2005; 7: 474-82.

66. Lukong KE, Richard S. Breast tumor kinase BRK requires kinesin- 2 subunit KAP3A in modulation of cell migration. Cell Signal 2008; 20: 432-42.

67. Benyamini P, Webster P, Meyer DI. Knockdown of p180 eliminates the terminal differentiation of a secretory cell line. Mol Biol Cell 2009; 20: 732-44.

68. Ogawa-Goto K, Tanaka K, Ueno T, et al. p180 is involved in the interaction between the endoplasmic reticulum and microtubules through a novel microtubule-binding and bundling domain. Mol Biol Cell 2007; 18: 3741-51.

69. Barbe L, Lundberg E, Oksvold P, et al. Toward a confocal subcellular atlas of the human proteome. Mol Cell Proteomics 2008; 7: 499-508.

70. Olsen JV, Blagoev B, Gnad F, et al. Global, in vivo, and site-specific phosphorylation dynamics in signaling networks. Cell 2006; 127: 635-48.
71. Savitz AJ, Meyer DI. 180-kD ribosome receptor is essential for both ribosome binding and protein translocation. J Cell Biol 1993; 120: 853-63.

72. Diefenbach RJ, Diefenbach E, Douglas MW, et al. The ribosome receptor, p180, interacts with kinesin heavy chain, KIF5B. Biochem Biophys Res Commun 2004; 319: 987-92.

73. Ueno $T$, Tanaka $K$, Kaneko $K$, et al. Enhancement of procollagen biosynthesis by p180 through augmented ribosome association on the endoplasmic reticulum in response to stimulated secretion. J Biol Chem 2010; 285: 29941-50.

74. Cardoso CM, Groth-Pedersen L, Hoyer-Hansen M, et al. Depletion of kinesin $5 \mathrm{~B}$ affects lysosomal distribution and stability and induces peri-nuclear accumulation of autophagosomes in cancer cells. PLoS One 2009; 4: e4424.

75. Krasnov GS, Oparina N, Khankin SL, et al. Colorectal cancer 2D-proteomics: identification of altered protein expression. Mol Biol (Mosk) 2009; 43: 348-56 (in Russian).

76. Rastogi $\mathrm{P}$, Anderson SJ, Bear HD, et al. Pathologic assessment of response to induction chemotherapy in breast cancer. Preoperative chemotherapy: updates of National Surgical Adjuvant Breast and Bowel Project Protocols B-18 and B-27. J Clin Oncol 2008; 10: 78-85.

77. Kuerer HM, Newman LA, Smith TL, et al. Clinical course of breast cancer patients with complete pathologic primary tumor and axillary lymph node response to doxorubicin-based neoadjuvant chemotherapy. J Clin Oncol 1999; 17: 460-9.

78. Symmans WS, Peintinger F, Hatzis C, et al. Measurement of residual breast cancer burden to predict survival after neoadjuvant chemotherapy. J Clin Oncol 2007; 25: 4414-22.

79. Zhang F, Chen J, Wang M, et al. A neural network approach to multi-biomarker panel discovery by high-throughput plasma proteomics profiling of breast cancer. BMC Proc 2013; 7: S10. 